How to reference this article

Maślanka-Soro, M. (2021). Il "tragico" disdegno di Farinata degli Uberti nel canto X dell'Inferno di Dante. Italica Wratislaviensia, 12(2), 149-168.

DOI: http://dx.doi.org/10.15804/IW.2021.12.2.08

\author{
Maria Maślanka-Soro \\ Uniwersytet Jagielloński w Krakowie, Polonia \\ maria.maslanka-soro@uj.edu.pl \\ ORCID: 0000-0002-0230-3836
}

\title{
IL “TRAGICO” DISDEGNO DI FARINATA DEGLI UBERTI \\ NEL CANTO X DELL'INFERNO DI DANTE
}

\author{
THE "TRAGIC" CONTEMPT OF FARINATA \\ DEGLI UBERTI \\ IN CANTO X OF DANTE'S INFERNO
}

\begin{abstract}
Dante's Inferno presents an essentially non-tragic view of reality based on the Christian concept of Man in his historical and eschatological aspect. Nonetheless, some of Dante's episodes, like the one of Farinata degli Uberti, appear to contain a certain element of tragedy because of the virtues marking the characters involved, which endow them with a certain nobility, giving rise to an air of tragedy. To examine the nature of this "tragic" quality, I shall invoke Erich Auerbach's concept of figural realism as applied to Dante's masterpiece. A character's life on earth is a prefiguration of his life after death, the fulfilment of his earthly existence concluding his earthly deeds. The soul's fate post mortem bespeaks the quintessence of its life, the tangible sign of which is its contrappasso. The chief conflict takes place between the character and his fulfilment, but it also generates further conflicts: between the soul's past on earth and its current condition in Hell; between the qualities that marked it in the past that could objectively be considered virtuous, and its current status amongst the damned, and others. Only in the eyes of sinners are these conflicts seen as tragic, but not from the point of view of Dante the Author, who discredits these conflicts with a variety of rhetorical and stylistic devices. I endeavour to explain the seemingly tragic quality in Farinata degli Uberti, one of the "magnanimous" spirits confined in Hell. At first glance he may seem reminiscent of the heroes of Greek tragedy, but on closer scrutiny his "magnanimity" takes on a sinister quality, and this is how Dante wants his readers to see the connection between Farinata's perverse political commitment verging on fanaticism, and his sin of heresy, to which Farinata seems to turn a blind eye.
\end{abstract}

Keywords: Dante, Inferno, Farinata degli Uberti, political fanaticism, heresy 
【1 termine "tragico", riferito ai dannati, richiede un chiarimento, in 1 quanto il poema dantesco, già a cominciare dal titolo ${ }^{1}$, pare estraneo a questa dimensione. Eppure alcuni episodi, tra cui quello di Farinata degli Uberti che si svolge nel canto X dell'Inferno, sembrano non privi di tragicità a causa di una certa nobiltà d'animo dei loro protagonisti e dello stile alto che rimane in sintonia con essa creando il clima tragico e rendendo più convincenti le parole dei personaggi i quali si comportano come se fossero protagonisti di una tragedia. Per indagare la vera natura di quel "tragico" ricorreremo al concetto del realismo figurale applicato da Erich Auerbach al capolavoro dantesco (ma inizialmente riferito dai Padri della Chiesa alla Bibbia), secondo cui gli eventi e i personaggi conservano nel mondo ultraterreno il loro senso storico o metastorico, diventando al tempo stesso veicoli della realtà universale ed eterna (Auerbach, 2007, pp. 176-226). Nella prospettiva escatologica la vita terrena è vista come prefigurazione di quella oltremondana che, a sua volta, si presenta come adempimento definitivo dell'altra. La poesia del "poema sacro" (Par. XXV 1) ${ }^{2}$ dovrebbe, sulla scia della Bibbia, far capire in che modo la nostra esistenza ${ }^{3}$ trova la sua piena dimensione nell'aldilà, in quanto tutti i fenomeni terreni hanno carattere unicamente figurativo. Di conseguenza, il senso letterale della Commedia - "status animarum post mortem" (Ep. XIII 24; cito da Alighieri, 2005) - esprimerebbe la quintessenza della nostra vita fungendo da sua forma perfectior il cui segno visivo è il contrappasso: il significato allegorico rivela non solo la qualità della pena in stretto rapporto con la colpa, come si evince dalla sua definizione nella stessa lettera a Cangrande (Ep. XIII 25), maprima di tutto - la verità su ogni essere umano fissata in eterno.

1 Nel Medioevo, quando si perde la nozione della teatralità dei generi drammatici antichi, per definire la tragedia e la commedia si prende in considerazione lo stile (alto per la tragedia, medio o basso per la commedia) e l'andamento dell'azione (dal lieto inizio alla funesta fine per la tragedia e l'inverso per la commedia); cf. Dante, De vulg. el. II IV 5-6; Idem, Ep. XIII 29-30. Del titolo dell'opera maggiore di Dante si sono occupati, tra gli altri, Tavoni (1998, pp. 9-34); Conte (2001, pp. 559-574).

2 Tutte le citazioni della Commedia sono tratte da Alighieri (2011).

3 Dante protagonista rappresenta, com'è risaputo, Dante storico e ogni uomo. 
La mimesis ${ }^{4}$ nell'Inferno consiste nella rappresentazione dei drammi umani che, come in una tragedia, sono destinati a non avere soluzione positiva. Essi si svolgono nelle coscienze delle anime dannate che, grazie alla memoria, sono "in possesso" di tutta la loro vita. Nell'esistenza immutabile dell'eterno presente irrompe incessantemente - tramite i ricordi - la realtà storica, cosicché la problematica fusione di entrambe le dimensioni - terrena e ultraterrena - porta con sé una lacerazione dialettica. Si tratta di un conflitto alimentato dalle passioni violente dei dannati che sono la continuazione di quelle responsabili dei loro drammi dopo la morte, con la differenza che la tensione accumulata non trova nessuno sbocco e contribuisce al loro tormento.

Il conflitto più significativo sarebbe quello tra la figura e il suo adempimento, vale a dire tra la vita terrena e la forma che l'anima è costretta ad assumere nell'aldilà, la quale si manifesta tramite il cosiddetto corpo aereo ${ }^{5}$, strumento di sofferenza fisica e spirituale. Questo conflitto ne genera altri: tra il passato terreno e il presente infernale, tra, da una parte, i valori oggettivamente positivi che appartengono a quel passato, come passione amorosa o politica, onore, desiderio di conoscenza e, dall'altra, l'attuale condizione dei dannati, tra la necessità soggettiva che li aveva spinti a sottomettere questi valori a fini puramente terreni, lontani dalle verità ultime, e la necessità oggettiva che ne risulta post mortem. Si pone la questione se i conflitti in questione sono tragici e la risposta non è semplice. Lo sono se vengono considerati dal punto di vista dei peccatori (e di Dante pellegrino, almeno nei primi cerchi infernali, dove la sua consapevolezza del male e la comprensione dell'opera della giustizia divina è ancora insufficiente e in continua evoluzione). Non lo sono, invece, se si assume la prospettiva di Dante autore il quale mette in discussione quei valori tramite l'uso di artifici retorico-stilistici

4 Con questo termine si indica (dai tempi di Aristotele) una rappresentazione fittizia della realtà, in cui la trasposizione di un sistema di segni in un altro avviene grazie al ragionamento analogico, tramite l'uso di varie strategie linguistiche o linguisticoretoriche.

5 Le fasi della sua formazione vengono spiegate, com'è noto, da Stazio nel Purg. XXV 88-99, nell'ambito della storia dell'anima razionale dalla sua comparsa nel corpo umano fino al suo destino dopo la morte. 
caratterizzanti i dannati e i discorsi che loro rivolgono a Dante pellegrino, decostruendo il tragico. Perciò ai detti conflitti si sovrappone anche quello tra Dante autore e Dante pellegrino che non rimane mai statico, ma tende a diminuire progressivamente con il procedere della narrazione fino al riconoscimento (da parte del pellegrino) della giustizia divina - alla luce della dottrina del libero arbitrio ${ }^{6}$ - il cui carattere "oggettivo" costituisce il perno della visione cristiana che sta alla base dell'intero poema.

Del tragico nella Commedia si può parlare, infatti, solo nei termini di un gioco illusorio in cui alcuni dei dannati cercano di trascinare Dante pellegrino. Una funzione significativa svolge qui la retorica sensu lato, la quale non si limita al livello verbale, ma comprende gesti, sguardi o varie reticenze. Essa si nutre, per così dire, del pathos nel senso aristotelico della parola, cioè della sofferenza, costringendo Dante pellegrino a una lotta continua con il sentimento di pietà ${ }^{7}$ e a volte anche con quello di paura, gli stessi che nella famosa definizione aristotelica della tragedia (Aristotele, Poet. 6, 1449b 24-29) ${ }^{8}$ si aspettano dal destinatario delle vicende tragiche (ibid., 14, 1453b 11-14) - nell'Inferno lo status del destinatario di quelle vicende spetta sia a Dante pellegrino che, al livello extratestuale, al lettore implicito - e dovrebbero sciogliersi in una katharsis finale che qui si riveste di un senso etico-conoscitivo: Dante pellegrino (e il lettore) dovrebbe comprendere - grazie agli incontri con

${ }^{6}$ Che la situazione dei dannati vada vista proprio in funzione della dottrina del libero arbitrio (a cui Dante autore dedica spazio soprattutto nei canti centrali del Purgatorio: XVI 67-81; XVIII 65-75), si dice esplicitamente nell'Ep. XIII, laddove viene spiegato il senso allegorico del poema (25). E proprio questo senso diventa fondamentale per la decostruzione di ciò che appare come tragico.

7 All'inizio del canto II dell'Inferno che funge da proemio a tutta la cantica, il viaggio dantesco viene chiamato "la guerra / sì del cammino e sì de la pietate" (Inf. II 4-5).

${ }^{8}$ Dante non conosceva la Poetica di Aristotele, ma ciò non esclude che alcuni dei personaggi dell'Inferno (soprattutto i "magnanimi") assomiglino ai protagonisti delle tragedie antiche che per lo Stagirita furono fonte delle sue riflessioni sulla tragedia come genere letterario. 
i dannati - la natura e il senso profondo del male per vincerlo in se stesso e non cedergli in futuro'.

La katharsis e il pathos sono dunque due categorie rilevanti tramite cui alcuni episodi presentano affinità con la tragedia antica. Un'altra potrebbe essere la peripeteia che coincide con il rovesciamento della situazione (da positiva in negativa), anche se non sempre fa parte dell'intreccio tragico. In Dante si osserva una svolta simile (anche se non così radicale) nell'episodio di Farinata, come vedremo nella parte analitica. Occorre però tener conto del fatto che, sebbene il pathos costituisca secondo Aristotele la condizione sine qua non di una situazione tragica (ibid., 14, 1453b 38-39), nel caso dei dannati esso perde questa sua funzione, in quanto per lo Stagirita la sofferenza di un uomo malvagio non dovrebbe suscitare né pietà né paura (ibid., 13, 1453a 1-4).

Il modo in cui i protagonisti di alcuni episodi dell'Inferno si presentano a Dante pellegrino, ancora non pienamente consapevole dei meccanismi più profondi della giustizia divina, a prima vista rassomiglia al modo di agire di un eroe tragico nel senso aristotelico della parola (ibid., 13, 1453a 7-10). Tale situazione riguarda i dannati che appartengono alla categoria dei cosiddetti magnanimi: Farinata degli Uberti, Ulisse, nonché le anime degli "spiriti magni”" che scontano la pena nel Limbo. La loro magnanimità, che corrisponde alla megalopsychia aristotelica (Forti, 1961, pp. 329-364, in particolare pp. 340 sgg.; Idem, 1977 , passim $)^{10}$ descritta nell'Etica nicomachea, rimane in funzione del tragico sopra delineato, dal momento che esiste in stretto rapporto con la colpa. Lo Stagirita la intende - contrapponendole la chaunotēs (presunzione) e la mikropsychia (pusillanimità) - come virtù morale che ha per oggetto "grandi cose" 11 , in quanto ogni megalopsychos è "colui

9 Sulla questione della katharsis tragica e katharsis dantesca si veda MaślankaSoro (2010, pp. 86-96).

10 Vista l'accezione negativa che il termine assume nella Commedia, non si può escludere l'influenza di Cicerone, Ovidio o Stazio: cf. Scott (1977, pp. 9-45); Consoli (2005, pp. 55-57).

11 Eth. Nic. IV 3, 1123a 34. Le citazioni dall'Etica Nicomachea sono tratte da Aristotele (1993). 
che si stima degno di grandi cose e lo è veramente"12. Virgilio (2010, p. 212) apprezza la magnanimità degli eroi che Enea incontra nei Campi Elisi, chiamandoli "magnanimi heroes" (Aen. VI, 307). Invece per autori cristiani come San Bernardo (Serm. in Cant. XVI, 10, cit. in Gauthier, 1947-1948, pp. 187-336) o Alberto Magno (Albertus Magnus, 1968, pp. 252-253), questa disposizione d'animo - divenuta sinonimo di superbia - assume un significato chiaramente negativo ${ }^{13}$.

Nella valutazione del Farinata dantesco che viene esplicitamente chiamato dal narratore "magnanimo" (Inf. X 73) e si distingue per una particolare "grandezza infernale", l'opinione dei critici rimane divisa. Ciò vale soprattutto per i giudizi passati, in quanto le interpretazioni relativamente recenti sono più riservate e oggettive per quanto concerne il suo significato e la funzione svolta nel contesto del canto. Alcuni parlano del "titanismo" del ghibellino di cui aveva dato prova nell'essersi opposto da solo alla distruzione di Firenze dopo la sanguinosa battaglia di Montaperti ${ }^{14}$. Un atteggiamento "sovrumano" egli dimostra, secondo gli stessi critici, pure nell'inferno sopportando fieramente la pena e mostrando il disprezzo per la realtà circostante: "el s'ergea col petto e con la fronte / com'avesse l'inferno a gran dispitto" (vv. 35-36). Altri, invece, criticano il suo "epicureismo" e la politica di fazione, pericolosa per l'equilibrio del comune fiorentino. Il primo filone è rappresentato da Francesco De Sanctis (1967, pp. 653-680), Ettore Paratore (1976,

12 Ibidem, IV 3, 1123 b 1-2; cf. Tommaso d'Aquino, In Eth. IV lect. 8, n. 736: "Ille videtur esse magnanimus qui dignum seipsum aestimat magnis, idest ut magna faciat et magna ei fiant, cum tamen sit dignus" (cito da Thomas Aquinas, 1949); Dante, Conv. IV XVII 5: "Magnanimitade, la quale è moderatrice e acquistatrice de' grandi onori e fama" (cito qui e altrove da Alighieri, 2019).

13 Ma già Cicerone o Sallustio avvertono nella magnanimitas una minaccia alle tradizionali virtù romane, nonché il pericolo che il magnus animus possa degenerare in superbus animus sotto la pressione dell'ambizione o dell'atteggiamento altezzoso (cf. ad es. Cicero, Ep. IV 9, 4) e un significato altrettanto negativo (di arroganza o tracotanza) compare in alcuni poeti noti a Dante, come Ovidio o Stazio in riferimento ai personaggi mitologici come Fetonte o Capaneo (vedi Ovidio, Met. II 111-114; Stazio, Theb. XI 1-2; cf. Scott (1977, pp. 15 sgg.).

$14 \mathrm{Su}$ questo fatto ritorneremo più avanti. 
pp. 215-255) e Daniele Mattalia ${ }^{15}$, il secondo - da Rocco Montano (1960, pp. 707-716) e Giorgio Padoan (1976, pp. 83-99), per citare solo alcuni dantisti di rilievo. Accanto a queste voci non mancano altre che prendono le distanze dalle analisi moralizzanti, cercando la funzione dell'episodio nel macrotesto dell'Inferno e nella sua dimensione poetica, senza trascurare il personaggio di Cavalcante dei Cavalcanti, anche lui portatore di significati non meno importanti di quelli veicolati da Farinata (cf. ad es. Chiavacci Leonardi, 1979, pp. 84 sgg.; Scott, 1977, pp. 49 sgg.; Dell'Aquila, 1988, pp. 61-83: alle pp. 61-63 cf. la bibliografia a questo canto; Giglio, 1990, pp. 65-104; Stäuble, 2000, pp. 139-150).

La varietà dei giudizi getta luce sulla complessità del carattere del ghibellino dantesco che sfrutta il colloquio con il pellegrino per creare la sua immagine, lontana dal ritratto lasciatoci dai contemporanei. Diversi suoi elementi, compresa la magnanimità (aristotelica), sembrano confermare la dimensione tragica del personaggio. Ma per Dante autore il ghibellino si presenta come un "nobile empio", uno di quelli che fin da sempre avevano "popolato" le carte dei poeti, a cominciare da Prometeo, il quale in Eschilo sconta la pena per aver violato l'ordine stabilito dal dio supremo; solo che nella seconda parte (per noi perduta) della dilogia il titano, dopo aver riconosciuto la sua hamartia, otteneva il perdono da parte di Zeus e riacquistava la libertà.

Il Farinata di Dante assomiglia piuttosto al suo Capaneo, punito tra i bestemmiatori con la pena del fuoco (affine a quella del ghibellino): dopo la morte è tale quale era durante la vita (Inf. XIV 51). Diversamente dal caso di Prometeo (e similmente alla condizione di tutti i dannati), il suo destino non può più mutare, ma ciò non significa che sia più tragico di quello del titano.

Fin dall'inizio colpisce che il dramma di Farinata, svoltosi in due "atti" divisi da un breve ma intenso "intermezzo" con Cavalcante, apparentemente non abbia legami con la sua condizione di peccatore destinato da Minosse al sesto cerchio popolato dai rappresentanti di varie eresie. Infatti, il nesso colpa/pena, fondamentale in una tragedia classica, passa (apparentemente, come vedremo) in secondo piano, come pure

15 Vedi D. Mattalia, commento a Inf. X 22, in Alighieri (1986, pp. 209-210). 
il conflitto tra il passato terreno e il presente infernale da cui, del resto, egli prende le distanze in ragione della sua magnanimità ${ }^{16}$, tutto assorto nel pensiero rivolto al passato - suo, della sua stirpe e della sua fazione (Inf. X 47). Ne nasce uno scontro drammatico con Dante, focalizzato sul conflitto tra ghibellini e guelfi che ebbe come effetto una serie di sconfitte e cacciate - a turno - degli uni o degli altri ${ }^{17}$ :

poi disse: "Fieramente furo avversi

a me e a miei primi e a mia parte,

sì che per due fiate li dispersi".

"S'ei fur cacciati, ei tornar d'ogne parte",

rispuos'io lui, "l'una e l'altra fiata;

ma i vostri non appreser ben quell'arte". (Inf. X 46-51)

La triplice ripetizione del pronome in prima persona nel v. 47 ("a me e a miei primi e a mia parte") è prova dell'egocentrismo di Farinata la cui passione politica è ben circoscritta e limitata agli interessi della propria fazione.

Nel dialogo con Dante sorprende l'assenza di qualsiasi cenno alle cause della sua condizione escatologica, il che potrebbe significare la totale mancanza di coscienza critica del ghibellino e tale atteggiamento corrisponderebbe alla hamartia aristotelica nel suo aspetto intellettuale (errore di valutazione $)^{18}$. Ma la problematica etica riemergerà in seguito e sarà connessa con il dramma personale del ghibellino, apparentemente solo politico.

Per il momento tale aspetto è assente (o offuscato), perché Farinata elude la verità sul male che aveva commesso come capo dei ghibellini e cerca di imporre la falsa visione di sé a Dante pellegrino grazie alle strategie retoriche e psicologiche, sforzandosi cioè di mostrare la propria superiorità assumendo un atteggiamento altezzoso nei confronti dell'in-

16 Vedi i già citati versi 35-36 del canto $\mathrm{X}$.

17 Si tratta delle vittorie dei ghibellini negli anni 1248 e 1260 (battaglia di Montaperti) e delle loro sconfitte negli anni 1258 e 1266 (battaglia di Benevento in cui perse la vita Manfredi, figlio di Federico II).

${ }_{18}$ Per i due aspetti del termine hamartia, morale e intellettuale, vedi Aristotele (2002, p. 158, nota 8). 
terlocutore ed esprimendosi in modo raffinato a cominciare dall'iniziale captatio benevolentiae $^{19}$. Il successivo scatto di aggressività (vv. 46-48) provoca una frecciata da parte di Dante pellegrino (vv. 49-51) che rivela allusivamente un fatto probabilmente sconosciuto ${ }^{20}$ a Farinata, in quanto avvenuto dopo la sua morte (1264): la definitiva cacciata dei ghibellini da Firenze e da altre città toscane dopo la grande sconfitta della parte ghibellina a Benevento nel 1266, seguita dall'esilio degli Uberti e dalla confisca dei loro beni. Quest'allusione provoca una reazione del ghibellino in cui colpisce il cambiamento di tono nella seconda parte del colloquio con Dante, ripreso dopo la scena con Cavalcante dei Cavalcanti - una brusca irruzione del padre di Guido, desideroso di sapere perché suo figlio non prende parte, assieme a Dante, al viaggio ultraterreno, secondo lui riservato a quelli che si distinguono per "altezza d'ingegno" (v. 59). Colpito nell'amor proprio (e quello per la sua fazione) Farinata tace e nel suo impietrirsi interiore e esteriore ${ }^{21}$ rimane insensibile al drammatico scambio di battute tra Dante e Cavalcante vibrante di forti emozioni:

Ma quell'altro magnanimo, a cui posta restato m'era, non mutò aspetto, né mosse collo, né piegò sua costa. (Inf. X 73-75)

Il contrasto con il comportamento del padre di Guido rafforza l'impressione della tragica statuaria immobilità del ghibellino, chiamato esplicitamente “magnanimo". Questo aspetto fa venire in mente l'antica

19 Farinata nel suo modus loquendi usa diversi latinismi e varie figure retoriche, ad es. la perifrasi: "la città del foco" (v. 22); latinismi: "parlando onesto" (v. 23), "la tua loquela" (v. 25), "molesto" (v. 27), "maggior tui" (v. 42); eufemismo: "questo letto" (v. 78); doppia perifrasi e inversione: "ma non cinquanta volte sia raccesa / la faccia de la donna che qui regge" (vv. 79-80) e altri.

20 Farinata fa sapere a Dante, verso la fine del loro colloquio (Inf. X 100-108), che i dannati (a conferma del fatto che lui pensa non solo agli "epicurei", cf. Inf. XIX 54; XXVII 28; XXXIII 122-123) non conoscono né il presente né il prossimo futuro (e tale sarebbe, infatti, l'anno 1266 rispetto a quello della sua morte nel 1264).

21 Aristotele (Eth. Nic. IV 3, 1124a 14-15) sottolinea che il magnanimo si mantiene impassibile di fronte alla sfortuna e alla fortuna. 
Niobe (Omero, Il. XXIV 602 sgg.; Ovidio, Met. VI, 146 sgg.) - che nella prima cornice del Purgatorio diventa l'esempio della superbia punita (Purg. XII 37) - o Prometeo il cui "eloquente" tacere nel prologo della tragedia eschilea esprime, oltre al dolore, il disprezzo nei confronti dei suoi tormentatori. Il silenzio che accentua la tensione creando una suspense (Gorzkowski, 2003, p. 122), viene infatti spesso sfruttato dai tragediografi antichi (cf. ad es. Winniczuk, 1951a, pp. 284-302; 1951b, pp. 359-376), in particolare da Sofocle, dove svolge una funzione drammatica, diventando un motivo quasi topico che precede il momento del suicidio da parte di un personaggio troppo provato dal destino ${ }^{22}$. D'altra parte, la reazione di Farinata fa venire in mente la svolta tragica, cioè la peripeteia che secondo Aristotele non può mancare in una perfetta tragedia (kalliste tragoidía), dove essa viene accompagnata dall'anagnorisis $^{23}$, dal riconoscimento cioè di una verità finora ignota al protagonista.

Fin dall'inizio del secondo "atto" di questo dramma l'atteggiamento di Farinata muta radicalmente: egli perde, a mio avviso solo apparentemente, la precedente altezzosità, aprendosi al dialogo. La sofferenza alla notizia dello scempio della sua fazione a Benevento coincide con la consapevolezza che la passione politica, culminata nella battaglia di Montaperti con la strage dei guelfi ${ }^{24}$, si è rivolta contro tutto ciò che gli era caro. Lo apprende da Dante in risposta al suo lamentarsi perché il popolo fiorentino si era rivelato così "empio" da perseguitare con vari decreti i suoi discendenti:

${ }_{22}$ Così reagisce Euridice (nell'Antigone) alla notizia della morte del figlio Emone, Deianira (nelle Trachinie) di fronte all'ingiusta accusa da parte del figlio Illo e Giocasta (nell'Edipo Re), dopo aver capito che Edipo era suo figlio.

${ }^{23}$ L'Edipo Re di Sofocle presenta una tale soluzione e sotto questo e altri aspetti merita, secondo Aristotele, il nome di tragedia modello: vedi ad es. Poet. 11, 1453a $32-33$.

${ }^{24}$ Questa strage fu così orribile che "tutte le strade e poggi e ogni rigo d'acqua pareva un grosso fiume di sangue" (A.M. Chiavacci Leonardi, commento a Inf. X 86, in Alighieri 2001, p. 186, dove si cita un'antica cronaca senese). 
"E se tu mai nel dolce mondo regge, dimmi: perché quel popolo è sì empio incontr' a' miei in ciascuna sua legge?".

Ond'io a lui: "Lo strazio e 'l grande scempio che fece l'Arbia colorata in rosso, tal orazion fa far nel nostro tempio". (Inf. X 82-87)

Si può notare che la passione politica di Farinata di cui egli vive nell'aldilà, fa quasi dimenticare, come si è accennato prima, il motivo per cui egli subisce la pena che corrisponde per analogiam alla sua colpa; eppure la descrizione verso la fine del canto IX e la spiegazione di Virgilio all'inizio del canto $\mathrm{X}$ non lasciano né Dante pellegrino né il lettore ignaro del fatto che assieme ad altri "epicurei" "che l'anima col corpo morta fanno" (Inf. X 15), il ghibellino è costretto a rimanere per l'eternità in una tomba infuocata ${ }^{25}$. Infatti in ciò consiste la sua poena sensus (come viene chiamato il contrappasso dai teologi medievali in opposizione alla cosiddetta poena damni ${ }^{26}$. D'altra parte, l'accento posto dallo stesso Farinata non lascia dubbi che il tormento causato dal suo accecamento politico si sia rivelato molto più doloroso della pena del fuoco infernale e tale verità soggettiva viene da lui esplicitamente ribadita nella ripresa del colloquio con Dante:

25 Colpisce l'ironia di questo contrappasso. Il fuoco potrebbe alludere, per antithesin, alla mancanza del "fuoco della fede" durante la vita. E il cimitero a cui viene paragonato lo spazio del sesto cerchio (Inf. IX 112-115), nell'immaginario dei lettori rende plasticamente l'idea dell'eternità della morte spirituale degli “epicurei”. Inoltre, la simbologia delle tombe infuocate fa venire in mente la morte sul rogo degli eretici, ma potrebbe anche alludere ironicamente alle credenze degli avverroisti (non rari tra gli "epicurei") che 1"“anima separata nullo modo patitur ab igne", dato che "finis terribilium est mors": vedi Scott (1977, pp. 32-33). Sulle varie sfumature del contrappasso in questo cerchio cf., tra gli altri, Gustarelli (1946, s.v. Eresiarchi); Padoan (1976, p. 94); Stäuble (2000, p. 140).

26 Su questi due tipi di punizione cui sono sottoposte le anime dell'Inferno (e quelle del Purgatorio) vedi Pasquazi (2005, pp. 390-392). 
"S'elli han quell'arte", disse, "male appresa,

ciò mi tormenta più che questo letto". (Inf. X 77-78)

Con l'eufemismo "letto" che indica la tomba infuocata in cui giace con altri, alzatosi solo momentaneamente per parlare con Dante, Farinata sminuisce la gravità della pena e quindi anche della colpa. Il suo attuale tormento, come si evince dai versi sopracitati, è di natura non morale, ma piuttosto psicologica. Nelle sue parole risuona il disprezzo verso la realtà infernale, notato già prima dal narratore (Inf. X 35-36). Non identificandosi con essa nella sua magnanimità, non capisce di farne parte e attribuisce il valore eterno e immutabile a ciò che appartiene alla storia, fissando nell'eterno presente la memoria delle cose passate e la sofferenza (celata) ad essa connessa.

Proprio per questo Farinata si riveste del tragico e l'epiteto "magnanimo" pare riferirsi soprattutto alla sua condizione psicologica nonostante in vari commenti si parli della grandezza morale del ghibellino ${ }^{27}$. Io credo che lo si possa considerare in questi termini solo se ci basiamo sull'accezione greca del magnanimo (megalopsychos), dove il valore dell'individuo è commisurato all'onore di cui egli gode nella società; di conseguenza, venuto meno quest'ultimo, la situazione volge inevitabilmente in tragico. Infatti, la sconfitta, non riscattata, dei ghibellini, è per Farinata un atto di umiliazione e ingiustizia e le sue conseguenze i decreti emessi contro la famiglia degli Uberti - prova dell'"empietà" del popolo fiorentino (v. 83). Spostando però il significato di quest'ultimo concetto per accusare altri, egli nega implicitamente che esso possa riferirsi a lui e attribuisce indirettamente il valore del sacrum agli atti sanguinosi e spietati che stanno dietro l'amore per la propria stirpe e per la patria (ghibellina!). Nelle sue parole riportate sopra (vv. 82-84) si può quindi leggere la conferma implicita e non intenzionale del peccato di eresia. Perciò - in risposta - Dante pellegrino chiama ironicamente

27 Vedi, a titolo di esempio, D. Mattalia, commento a Inf. X 36, in Alighieri (1986, p. 214). 
i detti decreti "orazioni" fatte nel "tempio" (vv. 85-87) ${ }^{28}$. In questo passo viene ribadito allusivamente, sfruttando il gioco di parole, uno stretto legame tra il peccato di eresia e il fanatismo politico. Ne viene fuori un'immagine di Farinata diversa da quella che egli vorrebbe lasciare di sé a Dante e - tramite lui - ai suoi contemporanei. Da questa situazione sorge il conflitto tra la figura e il suo adempimento, tra la necessità soggettiva che lui si era imposto indirizzando le proprie scelte verso degli pseudovalori, e quella oggettiva, intesa come effetto della giustizia divina, che si era compiuta dopo la morte.

La doppia dimensione della colpa di Farinata è stata notata da Francesco da Buti: "Fu messer Farinata del quale si dirà di sotto, d'avere inquietato e molestato la pace della sua patria con le sue sette, e divisioni" (1989, p. 282). La sua magnanimità include, accanto ai tratti aristotelici, quindi positivi, anche aspetti negativi, messi a fuoco già da alcuni autori cristiani che la fanno avvicinare alla superbia. Quelli aristotelici si riflettono nell' exploit eroico di cui Farinata fa menzione più avanti (vv. 91-93), dicendo che si era opposto da solo al Concilio di Empoli all'idea, approvata dai ghibellini di Toscana, di radere al suolo Firenze dopo la battaglia di Montaperti (G. Villani, Cronica VI 81). Tra i commentatori antichi prevale però, nel complesso, il giudizio negativo su Farinata e l'esplicita accusa di superbia, come si legge ad esempio nel già citato Francesco da Buti: "Questa magnanimità era in messer Farinata per vizio e non per virtù, cioè per superbia: imperò che in inferno non può essere virtù" (1989, p. 287). Tale contrapposizione era apparsa nel commento di Tommaso d'Aquino a un passo di Aristotele: "Praesumptio magnanimitati adversatur per excessum"29. Benvenuto da Imola non nutre dubbi che "fuit enim Farinata superbus cum tota sua stirpe" (1887, p. 337).

Queste e altre testimonianze confermano ciò che si può dedurre dal testo dantesco, vale a dire che la superbia era uno dei tratti principali del

28 Vedi A.M. Chiavacci Leonardi, commento a Inf. X 87, in Alighieri (2001, p. 186, dove la parola 'tempio' viene interpretata in senso proprio in quanto i consigli si tenevano in quei tempi nelle chiese cittadine).

29 Tommaso d'Aquino, Summa theol. II ${ }^{\mathrm{a}}$ II $^{\mathrm{ae}}$ 130, 2 (Thomas Aquinas, 2019-2020). 
carattere di Farinata, così in vita che in morte, dove la sua figura terrena trova il proprio adempimento.

Nella convinzione dei teologi medievali questo termine assunse un significato più negativo che in Aristotele, dove indicava uno degli stati d'animo estremi rispetto alla magnanimità ritenuta virtù. Rifacendosi alla Bibbia, essi indicano nella superbia il primum peccatum e la radice di tutti gli altri ${ }^{30}$, in quanto peccato di Lucifero. E, come spiega nel cielo di Giove l'Aquila composta dalle anime dei giusti, la colpa di questo "primo superbo" non consistette in un'aperta ribellione a Dio, ma nel rifiuto della luce della grazia: il Serafino ritenne per superbia di essere già perfectus e non solo potenzialmente tale (perfectabilis), come frutto non ancora maturo:

E ciò fa certo che 'l primo superbo, che fu la somma d'ogne creatura, per non aspettar lume, cadde acerbo. (Par. XIX 46-48) (11 $^{31}$

Un costante e convinto rifiuto dell'illuminazione tramite la grazia divina, proprio dei superbi, spetta al massimo grado agli eretici ${ }^{32}$. Lo conferma Pietro Alighieri, quando dice: "Quae superbia facit haeresim, non ignorantia" (1845, p. 130). Di un parere simile è Francesco da Buti secondo cui gli eretici stoltamente e con una forte ostinazione sono attaccati alle loro false opinioni: "L'eretico tiene sepolta la ragione nella sua falsa opinione, la quale è dura come la pietra" (1989, p. 272). Il loro errore più grande non è quello di nutrire idee sbagliate, ma di ostinarsi a permanere in questo stato, secondo il detto: "Errare humanum est, perseverare diabolicum" (Boccaccio, 1965, pp. 505, 507).

30 Vedi Syr X 13: "Initium omnis peccati superbia"; Tommaso d'Aquino, Summa theol. II $^{\text {a }} \mathrm{II}^{\text {ae }} 162,7$.

31 Vedi B. Garavelli, commento a Par. XIX 46-51, in Alighieri (1993, p. 285).

32 Il meccanismo di bloccare la conoscenza della verità in seguito a certi ragionamenti stolti e presuntuosi viene spiegato nel Paradiso dantesco da Tommaso d'Aquino (Par. XIII 115-120). 
Visti i legami tra la passione politica di Farinata (la quale nella forma estrema può portare al fanatismo) e la superbia, nonché tra quest'ultima e l'eresia, è logico pensare ad un'affinità tra la sua colpa politica e quella religiosa: la prima conduce a pericolose divisioni e conflitti nell'ambito della società, la seconda - nell'ambito della Chiesa.

All'inizio di questo canto Virgilio chiama i seguaci di Epicuro ${ }^{33}$ quelli "che l'anima col corpo morta fanno" (Inf. X 15). Nel medioevo, a partire dal secolo X, secondo la testimonianza di Alvarus de Cordova (Philippe, 1896, pp. 154 sgg.), il filosofo greco passava, mediante una semplificazione del suo complesso insegnamento, per precursore dei "materialisti", seguaci dell'eresia che negava l'immortalità dell'anima; accanto a questa, circolava un'altra opinione, dipendente da una fonte diversa, che Dante aveva conosciuto prima di scrivere il canto di cui ci stiamo occupando. Infatti, la caratteristica di Epicuro nel Convivio, basatasi sul giudizio di Cicerone espresso nel De finibus bonorum et malorum, differisce di molto dalla sua condanna nell'opera maggiore dove, l'unico tra i grandi filosofi antichi, non soggiorna nel Limbo, ma appunto nel cerchio degli eretici. Le cose stanno diversamente nel trattato filosofico dove vengono ricordate le opinioni dei rappresentanti delle tre grandi scuole di filosofia sulla natura del sommo bene a cui l'uomo dovrebbe tendere in vita. E così, secondo gli stoici, esso consiste nella "rigida onestate: cioè rigidamente, sanza respetto alcuno la verità e la giustizia seguire, di nulla mostrare dolore, di nulla mostrare allegrezza, di nulla passione avere sentore" (Conv. IV VI 9); invece per gli epicurei l'uomo dovrebbe cercare di raggiungere una particolare voluptade che corrisponde alla fuga da ogni dolore: "dicea che «voluptade» non era altro che «non dolore», sì come pare Tulio recitare nel primo di Fine di Beni" (Conv. IV vi 12). L'apparente incongruenza o contraddizione tra quello che leggiamo nel Convivio e nella Commedia è probabilmente dovuta al fatto che quando lavorava sul Convivio, Dante non sapeva ancora che Epicuro negava la vita dell'anima dopo la morte fisica, perché

33 Su Epicuro e sugli epicurei nell'Inferno di Dante vedi Stabile (2005, pp. 495-496; 489-495). Vedi anche il recente contributo di Villa (2020, pp. 356-374). 
il suo nome è implicitamente compreso nel contesto dell'aspra critica che egli fa di questa opinione:

Dico che intra tutte le bestialitadi quella è stoltissima, vilissima e dannosissima, chi crede dopo questa vita non essere altra vita; però che, se noi rivolgiamo tutte le scritture, sì de' filosofi come delli altri savi scrittori, tutti concordano in questo, che in noi sia parte alcuna perpetuale. (Conv. II VIII 8) ${ }^{34}$

Dalla messa in discussione della vita ultraterrena c'era solo un passo alla negazione del ruolo della giustizia divina, sia nei confronti dei buoni che dei cattivi e ciò poteva minare le regole della vita morale e dell'ordine universale.

Il cambiamento del giudizio da positivo a negativo può essere dovuto alla lettura delle Etimologie di Isidoro di Siviglia (Isidorus Hispalensis Episcopus, 1911, VIII vi 15-17), dove Epicuro viene accusato di non aver cercato la vera sapienza e i suoi immorali discepoli di essersi dati ai piaceri del corpo. Inoltre, si potrebbe citare l'operetta di Rabano Mauro, De universo, che presenta diverse affinità con le Etimologie: entrambi gli autori si trovano nel Paradiso di Dante, tra i sapienti (X 131; XII 139). Nella stessa prospettiva vengono visti gli "epicurei” fiorentini da Giovanni Villani nella Nuova Cronica (IV 30).

Per il Farinata dantesco il surrogato della felicità nella vita terrena doveva essere la non mai spenta e ossessivamente vissuta passione politica, strettamente legata all'interesse della sua casata e della sua fazione, passione che l'epicureismo rendeva priva di scrupoli di natura morale. Ma a prevalere è il dramma di un uomo costretto a constatare per l'eternità la fallacia delle proprie convinzioni filosofiche, rivelatesi una grande illusione, nonché la vanità della propria passione politica. Tale passione, che nel passato era stata per lui un valore supremo, diventa, nel presente della realtà infernale, fonte di eterna sofferenza a ricordo

${ }^{34}$ Gianfranco Fioravanti ritiene che dietro a quest'ultima affermazione stia un passo del De natura et origine animae, dove Alberto Magno afferma che tutte le scuole filosofiche sono concordi nell'affermare che l'anima umana sopravvive oltre la morte ed estende questa convinzione anche agli Epicurei: vedi G. Fioravanti, nota a Conv. II VIII 8, in Alighieri (2019, p. 190). 
della fine tragica di quello in cui egli aveva riposto tutte le speranze: la famiglia e il partito ghibellino ${ }^{35}$.

\section{BIBLIOGRAFIA}

Albertus Magnus (1968). Opera omnia. Muenster: Aschendorff.

Alighieri, D. (1986). La Divina Commedia (4th edition, vol. 1) (edited by D. Mattalia). Milano: BUR.

Alighieri, D. (1993). La Commedia (vol. 3) (edited by B. Garavelli under the supervision of M. Corti). Sonzogno: Bompiani.

Alighieri, D. (2001). Commedia (edited by A.M. Chiavacci Leonardi). Bologna: Zanichelli.

Alighieri, D. (2005). Le opere latine (edited by L. Coglievina, R.J. Lokaj, \& G. Savino). Roma: Salerno Editrice.

Alighieri, D. (2011). La Commedia (voll. 1-3) (edited by R. Hollander, translated by S. Marchesi). Firenze: Olschki.

Alighieri, D. (2019). Convivio (edited by G. Fioravanti \& [for Dante's “canzoni"] C. Giunta). Milano: Mondadori.

Alighieri, P. (1845). P. Allegherii super Dantis ipsius genitoris Comoediam Commentarium (edited by V. Nannucci). Florentiae: Piatti.

Aristotele. (1993). Etica Nicomachea, full Greek text alongside the Italian translation (edited by C. Mazzarelli). Milano: Rusconi.

Aristotele. (2002). Poetica (16th edition) (edited by D. Lanza). Milano: BUR. Auerbach, E. (2007). Studi su Dante, pref. D. Della Terza (translated by M.L. De Pieri Bonino, \& D. Della Terza) (3rd edition). Milano: Feltrinelli.

Benvenuto da Imola (1887). Comentum super Dantis Aldigherij Comoediam, vol. 1 (edited by I.Ph. Lacaita). Florentiae: Barbèra.

Boccaccio, G. (1965). Esposizioni sopra la Comedia di Dante (edited by G. Padoan). Milano: Mondadori.

Chiavacci Leonardi, A.M. (1979). La guerra de la pietate. Napoli: Liguori.

Consoli, D. (2005). Magnanimitate, Magnanimo. In Enciclopedia Dantesca, vol. 11 (pp. 55-57). Milano: Mondadori.

35 La fine tragica toccò pure ai resti mortali di lui e di sua moglie: nel 1283 sono stati riesumati e sparsi in una terra non consacrata, in seguito all'accusa postuma di eresia: vedi Frugoni (1966, p. 263). 
Conte, S. (2001). Le finalità del comico: una nuova proposta per l'interpretazione della intitulatio della "Commedia". Critica del testo, IV/3, 559-574.

D'Alfonso, R. (1982). "Comico" e "commedia": appunti sul titolo del poema dantesco. Filologia e critica, VII, 3-41.

Dell'Aquila, M. (1988). Il Canto X dell'Inferno. In L. Reina (Ed.), Humanitas e Poesia. Studi in onore di Gioacchino Paparelli (pp. 61-83). Salerno: Lavegli.

De Sanctis, F. (1967). Opere (2nd edition, vol. 5) (edited by S. Romagnoli). Torino: Einaudi.

Forti, F. (1977). Magnanimitade. Studi su un tema dantesco. Bologna: Pàtron.

Forti, F. (1996). Il Limbo dantesco e i megalopsichoi dell'Etica Nicomachea. Giornale storico della letteratura italiana, CXXXVIII, 329-364.

Francesco da Buti (1989). Commento sopra la Divina Commedia di Dante Alighieri, vol. 1 (edited by C. Giannini). Pisa: Nistri.

Frugoni, A. (1966). Il canto X dell'Inferno. In Nuove Letture Dantesche, vol. 1 (pp. 261-283). Firenze: Le Monnier.

Gauthier, R. (1947-1948). Trois commentaires averroïstes sur l'Ethique à Nicomaque. Archives d'Histoire Doctrinale et Littéraire du Moyen Age, XXII-XXIII, 187-336.

Giglio, R. (1990). Autore e lettori. Napoli: Lubrensi.

Gorzkowski, A. (2003). Tacent, laudant. Retoryka milczenia w traktacie Albertana z Brescii "Ars loquendi et tacendi". In T. Michałowska (Ed.), Mediewistyka literacka w Polsce (pp. 119-131). Warszawa: IBL.

Gustarelli, A. (1946). Eresiarchi. In A. Gustarelli (Ed.), Dizionario dantesco per lo studio della "Divina Commedia". Milano: Malfasi.

Isidorus Hispalensis Episcopus (1911). Etymologiarum sive originum libri XX, edited by W. M. Lindsay. Oxonii.

Kelly, H. A. (1993). Ideas and Forms of Tragedy from Aristotle to the Middle Ages. Cambridge: Cambridge University Press.

Maślanka-Soro, M. (2010). Tragizm w Komedii Dantego (2nd edition). Kraków: Universitas.

Montano, R. (1960). Per l'interpretazione del canto degli Epicurei. Convivium, n. s. $V I, 707-716$.

Padoan, G. (1976). Il canto X dell'Inferno. Letture classensi, V, 83-99.

Paratore, E. (1976). Il canto X dell'Inferno. Letture classensi, V, 215-255.

Pasquazi, S. (2005). Contrapasso. In Enciclopedia Dantesca, vol. 7 (pp. 389-392). Milano: Mondadori. 
Philippe, J. (1896). Lucrèce dans la théologie chrétienne du $\mathrm{III}^{\mathrm{e}}$ au XIII ${ }^{\mathrm{e}}$ siècle et spécialement dans les écoles carolingiennes. Revue de l'Histoire des Religions (Annales du Musée Guimet, Paris), XXXIII, 154 sgg.

Rossi, A. (1979). Il "serio-comico" dantesco. Paragone, XXX, 32-48.

Scott, J. A. (1977). Dante magnanimo. Firenze: Olschki.

Stabile, G. (2005). Epicurei. In Enciclopedia Dantesca, vol. 8 (pp. 489-495). Milano: Mondadori.

Stabile, G. (2005). Epicuro. In Enciclopedia Dantesca, vol. 8 (pp. 495-496). Milano: Mondadori.

Stäuble, A. (2000). Canto X. In G. Güntert, \& M. Picone (Eds.), Lectura Dantis Turicensis (vol. 1, 139-150). Firenze: Cesati.

Tavoni, M. (1998). Il titolo della "Commedia" di Dante. Nuova Rivista di Letteratura Italiana, $I / 1,9-34$.

Thomas Aquinas (1949). In decem libros Ethicorum Aristotelis ad Nicomachum exposition (edited by R. M. Spiazzi). Torino: Marietti.

Thomas Aquinas (2019-2020). Summa theologiae $\mathrm{II}^{\mathrm{a}} \mathrm{II}^{\mathrm{ae}}$ 130, 2. Retrieved from https://www.corpusthomisticum.org/sth3123.html

Winniczuk, L. (1951a). Milczenie jako element teatralny w dramacie starożytnym (1). Meander, 5-6, 284-302.

Winniczuk, L. (1951b). Milczenie jako element teatralny w dramacie starożytnym (2). Meander, 7-8, 359-376.

Villa, C. (2020). Ontologia di un fantasma. Epicuro eretico in 'Inf'., X. Rivista di studi danteschi, XX/3, 356-374.

Virgilio (2010). Eneide (translated by L. Canali, edited by E. Paratore, \& M. Beck). Milano: Mondadori.

Riassunto: L'Inferno dantesco presenta sostanzialmente una visione non tragica della realtà, basata sulla concezione cristiana dell'uomo nella sua dimensione storica ed escatologica. Eppure alcuni episodi, tra cui quello che si svolge nel canto $\mathrm{X}$ dell'Inferno, sembrano non privi di tragicità a causa di una certa nobiltà d'animo dei loro protagonisti e dello stile alto il quale rimane in sintonia con essa creando un clima tragico. Per indagare la vera natura di quel "tragico" ricorreremo al concetto del realismo figurale applicato da Erich Auerbach al capolavoro dantesco, secondo cui la vita terrena è prefigurazione di quella oltremondana che, a sua volta, si presenta come adempimento definitivo dell'altra. La condizione raggiunta dalle anime esprime la quintessenza della loro vita il cui segno visivo è il contrappasso. Il conflitto più significativo sarebbe quello tra la figura e il suo adempimento, ma esso ne genera altri: tra il passato terreno e il presente infernale, tra i valori oggettivamente positivi che appartengono a quel passato e l'attuale condizione dei dannati. I conflitti in questione sono tragici solo se considerati dal punto di vista dei peccatori, non lo sono invece dalla prospettiva di Dante autore il quale mette in discussione quei valori tramite l'uso di artifici retorico-stilistici caratterizzanti i dannati e i discorsi che loro rivolgono a Dante pellegrino, decostruendo così il tragico. Tenendo 
conto di queste considerazioni, si cerca di dimostrare in che cosa consiste il tragico apparente di Farinata degli Uberti, uno dei "magnanimi" infernali. A prima vista egli assomiglia agli eroi delle tragedie greche, ma un attento esame fa vedere come la sua magnanimità si riveste di un'accezione negativa e in questa prospettiva va visto il legame tra la sua passione politica, degenerata in fanatismo politico, e il peccato di eresia che egli sembra ignorare.

Parole chiave: Dante, Inferno, Farinata degli Uberti, fanatismo politico, eresia 